CERN-TH.6681/92

IEM-FT-61/92

FTUAM 92/31

\title{
SOFT SUSY BREAKING TERMS IN STRINGY SCENARIOS: COMPUTATION AND PHENOMENOLOGICAL VIABILITY
}

\author{
B. de CARLOS*, J.A. CASAS ${ }^{* *, *}$ and C. MUÑOZ*** \\ * Instituto de Estructura de la Materia (CSIC), \\ Serrano 123, 28006-Madrid, Spain \\ ** CERN, CH-1211 Geneva 23, Switzerland \\ *** Dept. de F!sica Teórica C-XI, \\ Univ. Autónoma de Madrid, E-28049 Madrid, Spain
}

\begin{abstract}
We calculate the soft SUSY breaking terms arising from a large class of string scenarios, namely symmetric orbifold constructions, and study its phenomenological viability. They exhibit a certain lack of universality, unlike the usual assumptions of the minimal supersymmetric standard model. Assuming gaugino condensation in the hidden sector as the source of SUSY breaking, it turns out that squark and slepton masses tend to be much larger than gaugino masses. Furthermore, we show that these soft breaking terms can be perfectly consistent with both experimental and naturalness constraints (the latter comes from the absence of fine tuning in the $S U(2) \times U(1)_{Y} \rightarrow U(1)_{e m}$ breaking process). This is certainly non-trivial and in fact imposes interesting constraints on measurable quantities. More precisely, we find that the gluino mass $\left(M_{3}\right)$ and the chargino mass $\left(M_{\chi^{ \pm}}\right)$ cannot be much higher than their present experimental lower bounds $\left(M_{3} \lesssim 285 \mathrm{GeV}\right.$; $\left.M_{\chi^{ \pm}} \lesssim 80 \mathrm{GeV}\right)$, while squark and slepton masses must be much larger $(\gtrsim 1 \mathrm{TeV})$. This can be considered as an observational signature of this kind of stringy scenarios. Besides, the top mass is constrained to be within a range $\left(80 \mathrm{GeV} \lesssim m_{t} \lesssim 165 \mathrm{GeV}\right.$ ) remarkably consistent with its present experimental bounds.
\end{abstract}

CERN-TH.6681/92

IEM-FT-61/92

FTUAM 92/31

October 1992 


\section{Introduction}

In the last two years there has been substantial progress in our understanding of supersymmetry (SUSY) breaking in string theories [1-11]. In particular, it has been learned that gaugino condensation effects in the hidden sector are able to break SUSY at a hierarchically small scale, at the same time as the dilaton $S$ and the moduli $T_{i}$ acquire reasonable vacuum expectation values $(\mathrm{VEVs})$. (The VEV of $S$ is related to the value of the gauge coupling constant, while those of $T_{i}$ define the size and shape of the compactified space.) This has been realized in the context of symmetric orbifold constructions. The modular invariance in the target space [3-6] as well as the presence of matter in the hidden sector play an important role in this process [2, 7-11]. On the other hand, the soft SUSY breaking terms in the observable sector are the phenomenological signature of any SUSY breaking mechanism. Therefore, it is essential to completely calculate the soft breaking terms of these scenarios and study their phenomenological viability. This is the motivation of the present paper.

There are two types of tests that the soft breaking terms should pass. First, they have to be consistent with the experimental (lower) bounds on gaugino masses, squark masses, etc. Second, they should be small enough not to spoil the SUSY solution to the gauge hierarchy problem, guaranteeing a successful $S U(2) \times U(1)_{Y} \rightarrow U(1)_{e m}$ breaking. The latter is a naturalness requirement. We find in this paper that SUSY breaking by gaugino condensation in the string models considered can be perfectly consistent with both tests. Moreover, this imposes very interesting constraints on the parameters defining the SUSY breakdown. As a consequence, we find that the gluino mass $\left(M_{3}\right)$ and the lightest chargino mass $\left(M_{\chi^{ \pm}}\right)$cannot be much higher than their present experimental lower bounds $\left(M_{3} \lesssim 285 \mathrm{GeV}, M_{\chi^{ \pm}} \lesssim 80 \mathrm{GeV}\right)$, while squark and slepton masses must be much larger $(\gtrsim 1 \mathrm{TeV})$. This can be considered as an experimental signature of this kind of models. Also, the top mass is constrained to be within a range $\left(80 \mathrm{GeV} \lesssim m_{t} \lesssim 165 \mathrm{GeV}\right)$ that is remarkably consistent with its present experimental bounds. In the calculation we have assumed minimal particle content in the observable sector and the vanishing of the cosmological constant. In section 2 we give the general form of the soft SUSY breaking terms for symmetric orbifold constructions. Interestingly enough, they show a certain lack of universality, unlike the usual assumptions of the minimal supersymmetric standard model. In order to get more quantitative results a specific SUSY breaking mechanism has to be considered. This is done in section 3 assuming the above mentioned gaugino condensation in the hidden sector (the only mechanism so far analized capable to 
generate a hierarchical SUSY breakdown in string constructions). In section 4 we confront the corresponding soft breaking terms with the two types of tests mentioned above. As a consequence we obtain the allowed ranges of variation for different physical quantities. Finally, in section 5 we present our conclusions.

\section{General characteristics}

Following the standard notation we define the soft breaking terms in the observable sector by

$$
\mathcal{L}=\mathcal{L}_{\mathrm{SUSY}}+\mathcal{L}_{\text {soft }}
$$

Here $\mathcal{L}_{\text {SUSY }}$ is the supersymmetric Lagrangian derived from the observable superpotential $W_{\text {obs }}$, which includes the usual Yukawa terms $W_{Y}$ and a mass coupling $\mu H_{1} H_{2}$ between the two Higgs doublets $H_{1}, H_{2}$. Assuming canonically normalized fields, $\mathcal{L}_{\text {soft }}$ is given by

$$
\mathcal{L}_{\text {soft }}=-\sum_{\alpha} m_{\alpha}^{2}\left|\phi_{\alpha}\right|^{2}-\frac{1}{2} \sum_{a=1}^{3} M_{a} \bar{\lambda}_{a} \lambda_{a}-\left(A m_{3 / 2} W_{Y}+B m_{3 / 2} \mu H_{1} H_{2}+\text { h.c. }\right)
$$

where $m_{3 / 2}$ is the gravitino mass, $\phi_{\alpha}$ represent the scalar components of the supersymmetric particles, and $\lambda_{a}$ are the $U(1)_{Y}, S U(2), S U(3)$ gauginos.

The characteristics of soft SUSY breaking terms in the observable sector are, to a great extent, determined by the type of $N=1$ SUGRA theory which appears in four

dimensions. As it is known, this is characterized by the gauge kinetic function $f$, the Kähler potential $K$ and the superpotential $W$. It is also customary to define $\mathcal{G}=K+$ $\log |W|^{2}$. These functions are determined, in principle, in a given compactification scheme, although in practice they are sufficiently well known only for orbifold compactification schemes [12, which on the other hand have proved to possess very attractive features from the phenomenological point of view [13]. More precisely, in the general case when the gauge group contains several factors $G=\prod_{a} G_{a}$, the exact gauge kinetic functions in string perturbation theory, up to small field-independent contributions, are [14-16]

$$
f^{a}=k^{a} S+\frac{1}{4 \pi^{2}} \sum_{i=1}^{3}\left(\frac{1}{2} b_{i}^{\prime a}-k^{a} \delta_{i}^{G S}\right) \log \left(\eta\left(T_{i}\right)\right)^{2}
$$

with

$$
b_{i}^{\prime a}=C\left(G^{a}\right)-\sum_{\Phi} T\left(R_{\Phi}^{a}\right)\left(1+2 n_{\Phi}^{i}\right)
$$


where the meaning of the various quantities appearing in eqs.(3), 4) is the following: $k^{a}$ is the Kac-Moody level of the $G^{a}$ group ( $k^{a}=1$ is a very common possibility), $S$ is the dilaton field, $T_{i}(i=1,2,3)$ are untwisted moduli whose real parts give the radii of the three compact complex dimensions of the orbifold $\left(\operatorname{Re} T_{i} \propto R_{i}^{2}, i=1,2,3\right), \delta_{i}^{G S}$ are 1-loop contributions coming from the Green-Schwarz mechanism, which have been determined for the simplest $(2,2) Z_{N}$ orbifolds [15]; $\eta\left(T_{i}\right)$ is the Dedekind function, $\Phi$ labels the matter fields transforming as $R_{\Phi}^{a}$ representations under $G^{a}$, and $n_{\Phi}^{i}$ are the corresponding modular weights. $C(G)$ denotes the Casimir operator in the adjoint representation of $G$ and $T(R)$ is defined by $\operatorname{Tr}\left(T^{i} T^{j}\right)=T(R) \delta^{i j}$. The Kähler potential $K$ is given by [17, 15]

$$
K=K_{o}\left(S, T_{i}\right)+\sum_{\alpha} K_{1}^{\alpha}\left|\phi_{\alpha}\right|^{2}+O\left(\left|\phi_{\alpha}\right|^{4}\right)
$$

where $\phi_{\alpha}$ are the matter fields and

$$
\begin{aligned}
K_{o}\left(S, T_{i}\right) & =-\log \left(S+\bar{S}+\frac{1}{4 \pi^{2}} \sum_{i=1}^{3} \delta_{i}^{G S} \log \left(T_{i}+\bar{T}_{i}\right)\right)-\sum_{i=1}^{3} \log \left(T_{i}+\bar{T}_{i}\right) \\
& \equiv-\log Y-\sum_{i=1}^{3} \log \left(T_{i}+\bar{T}_{i}\right) \\
K_{1}^{\alpha} & =\prod_{i}\left(T_{i}+\bar{T}_{i}\right)^{n_{\alpha}^{i}} .
\end{aligned}
$$

The function $Y$ can be considered [15] as the redefined gauge coupling constant (up to threshold corrections) at the unifying string scale $\left(Y=2 g_{\text {str }}^{-2}\right) . K_{o}$ is known at the 1-loop order, while $K_{1}^{\alpha}$ is only known at tree level. The complete dependence of $K$ (at tree level) on untwisted matter fields is also known [18], while for twisted fields this dependence has been conjectured in ref.[19]. However, for our purposes, i.e. studying soft breaking terms, the above expressions are sufficient. The 1-loop dependence on untwisted fields has also been conjectured in ref. 10

$$
K_{1-\text { loop }}^{\text {untw }}=-\log \left[S+\bar{S}+\frac{1}{4 \pi^{2}} \sum_{i} \delta_{i}^{G S} \log X_{i}\right]-\sum_{i=1}^{3} \log X_{i}
$$

where $X_{i}=\left(T_{i}+\bar{T}_{i}-\sum_{x}\left|A_{i}^{x}\right|^{2}\right)$, with $A_{i}^{x}$ denoting the untwisted fields (recall that untwisted fields carry a holomorphic index $i$ ). We will see below that (5) and (7) yield practically equivalent results. Finally, the perturbative superpotential $W^{p}$ at the renormalizable level has the form

$$
W^{p}=h_{I J K} \Phi_{I}^{(u)} \Phi_{J}^{(u)} \Phi_{K}^{(u)}+h_{I J K}^{\prime}\left(T_{i}\right) \Phi_{I}^{(t)} \Phi_{J}^{(t)} \Phi_{K}^{(t)}+h_{I J K}^{\prime \prime} \Phi_{I}^{(u)} \Phi_{J}^{(t)} \Phi_{K}^{(t)}
$$


where $\Phi_{I}^{(u)}\left(\Phi_{I}^{(t)}\right)$ are untwisted (twisted) charged matter fields. The value of $h_{I J K}, h_{I J K}^{\prime \prime}$ for the allowed couplings is simply a constant, while $h_{I J K}^{\prime}\left(T_{i}\right)$ are complicated but known functions of $T_{i}$ [20]. Besides $W^{p}$, there is a non-perturbative piece, $W^{n p}$, usually triggered by gaugino condensation effects in the hidden sector, which is crucial to break SUSY. In the following we will assume $\left\langle W^{p}\right\rangle=0,\left\langle W^{n p}\right\rangle \neq 0$, as it happens in all SUSY breaking scenarios so far analysed. $W^{n p}$ depends on the $S$ and $T$ fields and, sometimes, on certain matter fields $A$, which are singlet under the relevant gauge groups [7-11]. As has been shown [21, 11], the condition $\partial W / \partial A=0$ is the correct one to integrate out these fields. In consequence, we can use

$$
W^{n p}=W^{n p}\left(S, T_{i}\right)
$$

without any loss of generality. Expressions (3 -9) are to be understood at the string scale $M_{\text {Str }}=0.527 \times g \times 10^{18} \mathrm{GeV}$ [14], where $g \simeq 1 / \sqrt{2}$ is the corresponding value of the gauge coupling constant.

The next step is to calculate, once SUSY breaking is assumed, the form of the soft breaking terms based on eqs.(3 9). Some work in this direction has already been done. The gaugino masses were evaluated in ref. 3] for $\delta_{i}^{G S}=0$, and in ref. 22 for $\delta_{i}^{G S} \neq 0$ and a generic form of the Kähler potential. Likewise, the scalar masses when $\delta_{i}^{G S}=0$ were calculated in ref.[22], while the form of trilinear terms for $\delta_{i}^{G S}=0$ was given in ref. [6]. It is interesting to note that $\delta_{i}^{G S} \neq 0$ turns out to be crucial for a viable phenomenology, as will be seen in the next sections. In this paper we have calculated the general expressions for all the soft breaking terms. The notation used is that of ref. [23]. We work with the usual overall modulus simplification $T=T_{1}=T_{2}=T_{3}$.

\section{Gaugino masses:}

Physical gaugino masses $M_{a}$ for the canonically normalized gaugino fields $\left(\lambda_{\text {phys }}=\right.$ $\left.(\text { Re f })^{1 / 2} \lambda\right)$ are given by $\frac{1}{2}(\operatorname{Re} f)^{-1} \sum_{\alpha} \mathrm{f}_{\mathrm{a}}^{\alpha} \mathrm{e}^{\mathcal{G} / 2}\left(\mathcal{G}^{-1}\right)_{\alpha}^{\bar{\beta}} \mathcal{G}_{\bar{\beta}}$. Using the renormalization group (RG) equation for $M_{a}$, i.e. $M_{a}(Q)=\frac{\alpha_{a}(Q)}{\alpha_{a}\left(M_{S t r}\right)} M_{a}\left(M_{S t r}\right)$, and $\operatorname{Re} \mathrm{f}_{\mathrm{a}}=\mathrm{g}_{\mathrm{a}}^{-2}\left(\mathrm{M}_{\mathrm{Str}}\right)=$ $\left[4 \pi \alpha_{\mathrm{a}}\left(\mathrm{M}_{\mathrm{Str}}\right)\right]^{-1}$, we directly write $M_{a}$ at any $Q$ scale

$$
\begin{aligned}
M_{a}(Q) & =2 \pi \alpha_{a}(Q) m_{3 / 2}\left\{k^{a} Y^{2}\left(-\frac{1}{Y}+\frac{\bar{W}_{\bar{S}}}{\bar{W}}\right)+\frac{Y(T+\bar{T})^{2}}{3 Y+\frac{\delta^{G S}}{4 \pi^{2}}} \frac{\hat{G}_{2}(T)}{\pi}\right. \\
& \left.\times\left(\sum_{i} \frac{b_{i}^{\prime a}}{16 \pi^{2}}-k^{a} \frac{\delta^{G S}}{8 \pi^{2}}\right)\left[\frac{1}{T+\bar{T}}\left(3+\frac{\delta^{G S}}{4 \pi^{2}} \frac{\bar{W}_{\bar{S}}}{\bar{W}}\right)-\frac{\bar{W}_{\bar{T}}}{\bar{W}}\right]\right\},
\end{aligned}
$$

where $W_{\phi}=\partial W / \partial \phi, \phi=S, T, m_{3 / 2}=e^{K / 2}|W|, \delta^{G S}=\sum_{i} \delta_{i}^{G S}$ and $\hat{G}_{2}(T)=-\left(\frac{2 \pi}{T+T}+\right.$ $\left.4 \pi \eta^{-1} \frac{\partial \eta}{\partial T}\right)$. Furthermore it is worth mentioning that the factors $\sum_{i} b_{i}^{\prime a} \hat{G}_{2}(T)$ in (10) come 
from replacing $\sum_{i} b_{i}^{\prime a} G_{2}(T)$ factors (with $G_{2}(T)=\hat{G}_{2}(T)+\frac{2 \pi}{T+T}$ ) in the original expression. This counts the radiative contribution of the massless fields of the theory, and has to be performed in order to maintain the modular invariance of $M_{a}$ [22].

\section{Scalar masses:}

Scalar masses $m_{\tilde{\phi}_{\alpha}}$ are obtained from the scalar potential $V=e^{\mathcal{G}}\left(\mathcal{G}_{\alpha}\left(\mathcal{G}^{-1}\right)_{\beta}^{\bar{\alpha}} \mathcal{G}^{\bar{\beta}}-\right.$ 3). They have to be normalized by taking into account that the $\phi_{\alpha}$ kinetic terms are $\left(K_{\alpha \bar{\beta}}\right) D \phi_{\alpha} D \bar{\phi}_{\beta}$. Thus we obtain

$$
m_{\phi_{\alpha}}^{2}=V_{o}+m_{3 / 2}^{2}\left[1+\frac{n_{\alpha} Y^{2}}{\left(3 Y+\frac{\delta^{G S}}{4 \pi^{2}}\right)^{2}}\left|3+\frac{\delta^{G S}}{4 \pi^{2}} \frac{W_{S}}{W}-(T+\bar{T}) \frac{W_{T}}{W}\right|^{2}\right],
$$

where $V_{o}=\langle V\rangle$ is simply the value of the cosmological constant, which we will assume to vanish through the paper. One-loop corrections to the previous expressions are, in all probability, quite small].

\section{Trilinear scalar terms:}

If the perturbative superpotential contains a term $h \Phi_{1} \Phi_{2} \Phi_{3}$, then a trilinear scalar term appears in the effective Lagrangian after SUSY breaking. It has the form

$$
\begin{aligned}
\mathcal{L}_{\text {tril }} & =-m_{3 / 2} A \hat{h} \phi_{1} \phi_{2} \phi_{3}+\text { h.c. } \\
& =-m_{3 / 2}\left\{\left(1-Y \frac{\bar{W}_{\bar{S}}}{\bar{W}}\right)+\frac{Y}{3 Y+\frac{\delta^{G S}}{4 \pi^{2}}}\right. \\
& \times\left(3+\frac{\delta^{G S}}{4 \pi^{2}} \frac{\bar{W}_{\bar{S}}}{\bar{W}}-(T+\bar{T}) \frac{\bar{W}_{\bar{T}}}{\bar{W}}\right)\left(3+\sum_{\alpha=1}^{3} n_{\alpha}\right) \\
& \left.-\frac{Y(T+\bar{T})}{3 Y+\frac{\delta^{G S}}{4 \pi^{2}}}\left(3+\frac{\delta^{G S}}{4 \pi^{2}} \frac{\bar{W}_{\bar{S}}}{\bar{W}}-(T+\bar{T}) \frac{\bar{W}_{\bar{T}}}{\bar{W}}\right) \frac{h_{T}}{h}\right\} \hat{h} \phi_{1} \phi_{2} \phi_{3}+\text { h.c. },
\end{aligned}
$$

where $h_{T} \equiv \partial h / \partial T, \phi_{\alpha}$ are the (properly normalized) scalar components of the respective superfields, $n_{\alpha}$ are the corresponding modular weights, and $\hat{h}=e^{K / 2} \prod_{\alpha=1}^{3}\left(K_{\alpha \bar{\alpha}}\right)^{-1 / 2} h$ is the effective Yukawa coupling between the physical fields. Notice that for the untwisted case $\left[n_{\alpha}=-1, h \neq h(T)\right]$ the previous expression is drastically simplified.

\section{Bilinear scalar terms:}

It is not clear by now where a bilinear term of the form $\mu H_{1} H_{2}$ in $W_{\text {obs }}$ has its origin, although it is well known that this term is necessary in order to break $S U(2) \times U(1)_{Y}$

\footnotetext{
${ }^{1}$ For instance, scalar masses for untwisted fields computed from the conjecture of eq.(17) are given by $m_{\phi}^{2}=V_{o}+m_{3 / 2}^{2}\left[1-\frac{Y}{(3 Y+a)^{2}}\left(Y+\frac{a^{2}}{3 Y+a}\right)\left|3+a \frac{W_{S}}{W}-(T+\bar{T}) \frac{W_{T}}{W}\right|^{2}-\frac{1}{3 Y+a}\left(1+\frac{3 Y}{3 Y+a}\right) a\left|Y \frac{W_{S}}{W}-1\right|^{2}\right]$, with $a=\frac{\delta^{G S}}{4 \pi^{2}}$. Numerically, this amounts to $1 \%$ departure from the result of eq.(11).
} 
successfully․ This is the so-called $\mu$ problem. Assuming that this term is actually present, the corresponding bilinear term in the scalar Lagrangian turns out to be

$$
\begin{aligned}
\mathcal{L}_{b i l} & =-m_{3 / 2} B \hat{\mu} H_{1} H_{2}+\text { h.c. } \\
& =-m_{3 / 2} Y\left\{-\frac{\bar{W}_{S}}{\bar{W}}+\frac{3+n_{1}+n_{2}}{3 Y+\frac{\delta^{G S}}{4 \pi^{2}}}\left(3+\frac{\bar{W}_{\bar{S}}}{\bar{W}} \frac{\delta^{G S}}{4 \pi^{2}}-\frac{\bar{W}_{\bar{T}}}{\bar{W}}(T+\bar{T})\right)\right. \\
& \left.-\frac{(T+\bar{T})}{3 Y+\frac{\delta^{G S}}{4 \pi^{2}}}\left[3+\frac{\delta^{G S}}{4 \pi^{2}} \frac{\bar{W}_{\bar{S}}}{\bar{W}}-\frac{\bar{W}_{\bar{T}}}{\bar{W}}(T+\bar{T})\right] \frac{\mu_{T}}{\mu}\right\} \hat{\mu} H_{1} H_{2}+\text { h.c. }
\end{aligned}
$$

with a notation similar to that of eq.(12). Again, $\hat{\mu}=e^{K / 2} \prod_{\alpha=1}^{2}\left(K_{H_{\alpha} \bar{H}_{\alpha}}\right)^{-1 / 2} \mu$ is the effective parameter giving, for example, the coupling between the two higgsinos. For simplicity in the notation, we will drop the hat in $\hat{\mu}$ as well as in $\hat{h}$ from here on. Because of the above mentioned ignorance about the origin of $\mu$, we prefer in the following to consider $B$ as an unknown parameter.

A preliminary conclusion at this stage is that some of the common assumptions of the minimal supersymmetric standard model, in particular universality for all the gaugino masses, scalar masses and trilinear terms, do not hold in general (this was already noted in ref. 22]). Nevertheless, it is clear that eqs.(10-13) are not completely significant unless we have a way to evaluate $m_{3 / 2},\langle W\rangle,\left\langle W_{S}\right\rangle$, etc. This can only be done in the framework of a SUSY breaking scenario, by minimizing the corresponding scalar potential. We turn to this point in the next section.

\section{Realization of SUSY breaking by gaugino conden- sation}

As was mentioned above, the only mechanism so far analysed, capable of generating a hierarchical SUSY breakdown in superstring theories, is gaugino condensation in the hidden sector [26]. An extensive study of the properties of this mechanism can be found in ref. 11. Let us briefly summarize here the main characteristics. For a hidden sector gauge group $G=\prod_{b} G_{b}$, gaugino condensation induces a non-perturbative superpotential

$$
W^{n p}=\sum_{b} d_{b} \frac{e^{-3 k_{b} S / 2 \beta_{b}}}{[\eta(T)]^{6-\left(3 k_{b} \delta^{G S} / 4 \pi^{2} \beta_{b}\right)}},
$$

\footnotetext{
${ }^{2} \mathrm{~A}$ possible origin for $\mu$ has been proposed in ref. 24]. A trilinear term of the form $\mathrm{NH}_{1} \mathrm{H}_{2}, \mathrm{~N}$ being an $S U(3) \times S U(2) \times U(1)_{Y}$ singlet with non-vanishing expectation value could also do the job 25.
} 
where $\beta_{b}$ are the corresponding beta functions and $d_{b}$ are constants. Notice that $W_{T}^{n p}=$ $-2 \eta^{-1} \frac{\partial \eta}{\partial T}\left(3 W^{n p}+\frac{\delta^{G S}}{4 \pi^{2}} W_{S}^{n p}\right)$, which can be used to eliminate $W_{T}$ in (10-13). In particular,

$$
\begin{aligned}
M_{a}(Q) & =2 \pi \alpha_{a}(Q) m_{3 / 2}\left\{k^{a} Y^{2}\left(-\frac{1}{Y}+\frac{\bar{W}_{\bar{S}}}{\bar{W}}\right)-\frac{Y(T+\bar{T})^{2}}{3 Y+\frac{\delta^{G S}}{4 \pi^{2}}} \frac{\left|\hat{G}_{2}(T)\right|^{2}}{2 \pi^{2}}\right. \\
& \left.\times\left(\sum_{i} \frac{b_{i}^{\prime a}}{16 \pi^{2}}-k^{a} \frac{\delta^{G S}}{8 \pi^{2}}\right)\left(3+\frac{\delta^{G S}}{4 \pi^{2}} \frac{\bar{W}_{\bar{S}}}{\bar{W}}\right)\right\} \\
m_{\phi_{\alpha}}^{2} & =m_{3 / 2}^{2}\left[1+n_{\alpha}\left(\frac{T+\bar{T}}{2 \pi}\right)^{2}\left|\hat{G}_{2}(T)\right|^{2}\left|\frac{3+\frac{\delta^{G S}}{4 \pi^{2}} \frac{W_{S}}{W}}{3+\frac{\delta^{G S}}{4 \pi^{2}} \frac{1}{Y}}\right|^{2}\right]
\end{aligned}
$$

There is a large class of scenarios for which SUSY is spontaneously broken, yielding reasonable values of $m_{3 / 2}$ and $\langle Y\rangle$ without the need of fine tuning [11. A common ingredient of these models is the existence of more than one condensing group in the hidden sector [27, 1, 2]. The values of $m_{3 / 2}$ and $\langle Y\rangle$ turn out to depend almost exclusively on which the gauge group and matter content of the hidden sector are. Practically, any values of $m_{3 / 2}$ and $\langle Y\rangle$ are available by appropriately choosing the hidden sector. Consequently, we can consider $m_{3 / 2}$ and $Y$ as free parameters since no dynamical mechanism is already known to select a particular string vacuum. On the other hand, both $m_{3 / 2}$ and $\langle Y\rangle$ are almost independent of the value of $\delta^{G S}$ [11]. Likewise, the value of $\langle T\rangle$ turns out to be $\sim 1.23$ in all cases [3, 11]. This stability does not hold, however, for the other quantities $\left(\langle W\rangle,\left\langle W_{S}\right\rangle\right.$, etc.) appearing in eqs.(10-13) and (15,??). For example, as was pointed out in ref.[3], the combination $Y W_{S}-W$ (which appears in several places in the previous equations) is vanishing in the minimum of the scalar potential for $\delta^{G S}=0$, but this is no longer true for $\delta^{G S} \neq 0$. In this case, however, the scalar potential is much more involved, so a numerical analysis is in general necessary.

Fortunately, the results admit quite simple and useful parametrizations describing them very well (within $1 \%$ of accuracy). Next, we give these parametrizations for the common $k^{a}=1$ case. In particular, for the scalar masses

$$
m_{\phi_{\alpha}}^{2}=m_{3 / 2}^{2}\left[1+n_{\alpha}\left(0.078-1.3 \times 10^{-4} \delta^{G S}\right)\right] .
$$

It is really remarkable here the lack of dependence of (17) on the specific type of hidden sector considered. Actually, the influence of the hidden sector is completely summarized in the value of $m_{3 / 2}$. Moreover, the influence of the actual values of the modular weights $n_{\alpha}$ for the observable particles is quite small. 
On the contrary, the absolute value of $A$, i.e. the parameter defining the trilinear scalar terms in (12), has a stronger dependence on the modular weights of the relevant fields. In particular, if the three fields under consideration are untwisted, i.e. $n_{1}=n_{2}=$ $n_{3}=-1, A$ turns out to be very small in all the cases

$$
A^{\text {untw }} \lesssim 10^{-3}<<1
$$

(if $\delta^{G S}=0, A^{\text {untw }}=0$ exactly). For twisted fields a good parametrization is

$$
A=A^{u n t w}+\left(0.28-2.3 \times 10^{-4} \delta^{G S}\right)\left(3+\sum_{\alpha=1}^{3} n_{\alpha}\right)+\left(0.69-6.9 \times 10^{-4} \delta^{G S}\right) \frac{h_{T}}{h},
$$

where the precise value of $h_{T} / h$ depends on the specific Yukawa coupling considered (see ref. 20]). In general $h_{T} / h$ is negative and $\left|h_{T} / h\right| \lesssim O(1)$.

Concerning gaugino masses, it is worth noticing that for $\delta^{G S}=0$ the first term of eq.(15) (proportional to the $S \mathrm{~F}$-term) vanishes in the minimum of the potential [3]. Then the resulting gaugino mass is very tiny (e.g. for all the observable matter in the untwisted sector the gluino mass is $\left.M_{3}=m_{3 / 2} / 445\right)$. This is not surprising since it is generated thanks to the one-loop threshold correction of eq.(3). Fortunately, this fact does not hold for $\delta^{G S} \neq 0$. The values of the gaugino masses present a certain dependence on the type of orbifold considered. Consider first the case of the $Z_{3}$ and $Z_{7}$ orbifolds. For these schemes the threshold contributions to the $f$ function [see eq.(3)] are known to vanish [14] and the following equality holds for all the gauge group factors

$$
\sum_{i} b_{i}^{\prime a}-2 k^{a} \delta^{G S}=0
$$

As a consequence, there is a cancellation of the second term of eq.(15). The value of the first term at the minimum of the potential is easily parametrizable in the form

$$
M_{a}(Q)=-\alpha_{a}(Q) m_{3 / 2}\left(0.0120 \delta^{G S}+0.0029\right) .
$$

Hence, for the $Z_{3}$ and $Z_{7}$ orbifolds the value of $M_{a}$ is completely given in terms of $m_{3 / 2}$ and $\delta^{G S}$. For the rest of the $Z_{N}$ orbifolds things are different since eq. (20) is no longer true. Then, an equation similar to eq.(21) can be written in terms of $m_{3 / 2}, \delta^{G S}$ and $\sum_{i} b_{i}^{\prime a}$. Let us note, however, that for all the remaining $Z_{N}$ orbifolds (except for the $Z_{6}-\mathrm{II}$ ) the cancellation (20) still takes place for two of the three compactified dimensions], i.e.

$$
b_{i}^{\prime a}-2 k^{a} \delta_{i}^{G S}=0, \quad i=1,2 .
$$

\footnotetext{
${ }^{3}$ For the $Z_{6}-$ II case the cancellation holds in one complex dimension. On the other hand, the $Z_{6}-\mathrm{II}$ orbifold is one of the less interesting orbifolds from the phenomenological point of view [28].
} 
Roughly speaking, our numerical results indicate that parametrization (21) is still valid in these cases within a $30 \%$ error. As will be seen in the next section, this is enough for our purposes. Finally, as explained above, we choose to leave the value of $B$ [see eq.(13)] free, owing to our ignorance of the origin of $\mu$. Equations (17, 19,21) summarize the input of soft breaking terms to be studied from the phenomenological point of view. Before entering in this analysis, let us comment two features relative to eqs.(17,21). First, an interesting $m_{3 / 2}$-independent relation between $M_{a}$ and $m_{\phi_{\alpha}}$ can be obtained

$$
\frac{M_{a}^{2}\left(M_{Z}\right)}{m_{\phi_{\alpha}}^{2}\left(M_{S t r}\right)}=\frac{\alpha_{a}^{2}\left(M_{Z}\right)\left(0.0120 \delta^{G S}+0.0029\right)^{2}}{1+n_{\alpha}\left(0.078-1.3 \times 10^{-4} \delta^{G S}\right)} .
$$

Of course, in order to get the physical scalar masses, an RG running until the electroweak scale has to be performed. However, it is already clear that gaugino masses tend to be much smaller than scalar masses, since typically $\delta^{G S} \lesssim 50$ [15]. Second, as was mentioned in section $2, M_{a}$ and $m_{\phi_{\alpha}}^{2}$, as well as the trilinear terms, clearly show a certain lack of universality, unlike the usual assumptions of the minimal supersymmetric standard model.

\section{Phenomenological viability of the soft breaking terms}

\subsection{Experimental constraints}

As was mentioned in the introduction, there are two types of constraints on soft breaking terms: observational bounds and naturalness bounds. The first ones mainly come from direct production of supersymmetric particles in accelerators. This gives the following lower bounds on supersymmetric particle masses, as reported by the Particle Data Group 29

$$
\begin{array}{ll}
M_{3}>79 \mathrm{GeV}, & \mathrm{M}_{\chi^{ \pm}}>45 \mathrm{GeV} \\
m_{\tilde{q}}>74 \mathrm{GeV}, & \mathrm{m}_{\tilde{1}} \gtrsim 45 \mathrm{GeV}
\end{array}
$$

where $M_{3}$ is the gluino mass, $\chi^{ \pm}$is the lightest chargino, and $\tilde{q}, \tilde{l}$ collectively denote squarks and sleptons respectivelyf. A more stringent lower bound for the gluino and squark masses $(106 \mathrm{GeV})$ arises if $m_{\tilde{q}}=M_{3}$, which certainly is not the case here. The chargino bound is subjected to the condition $m_{\chi^{o}}<28 \mathrm{GeV}$ with $\chi^{o}$ the lightest neutralino, thus this bound does not necessarily apply. The above experimental bounds are to be confronted with the

\footnotetext{
${ }^{4}$ Less conservative bounds have been reported elsewhere 30 . The corresponding modification of our final results is completely straightforward.
} 
theoretical values from the SUSY breaking scenario we are analyzing. In particular, the gluino mass is simply given by eq.(21) evaluated at the $M_{Z}$ scale

$$
M_{3} \simeq \alpha_{3}\left(M_{Z}\right) m_{3 / 2}\left(0.0120 \delta^{G S}+0.0029\right)
$$

On the other hand, charginos (i.e. winos $\tilde{W}^{ \pm}$and higgsinos $\tilde{H}^{ \pm}$) mix via a $2 \times 2$ mass matrix whose lowest eigenvalue is given by

$$
\begin{aligned}
M_{\chi^{ \pm}} & =\frac{1}{2}\left[M_{2}^{2}+\mu_{R}^{2}+2 M_{W}^{2}\right. \\
& \left.-\sqrt{\left(M_{2}^{2}-\mu_{R}^{2}\right)^{2}+4 M_{W}^{4} \cos ^{2} 2 \beta+4 M_{W}^{2}\left(M_{2}^{2}+\mu_{R}^{2}+2 M_{2} \mu_{R} \sin 2 \beta\right)}\right]
\end{aligned}
$$

where $\operatorname{tg} \beta=\left\langle H_{2}\right\rangle /\left\langle H_{1}\right\rangle, \mu_{R}$ is the renormalized value of $\mu$, and $M_{2}$ is given by eq.(21). $M_{2}$ and $\mu_{R}$ are evaluated at the $M_{Z}$ scale. In all these equations the current central experimental values of $\alpha_{a}\left(M_{Z}\right)$ can be used

$$
\alpha_{1}\left(M_{Z}\right)^{e x p}=0.01693, \quad \alpha_{2}\left(M_{Z}\right)^{e x p}=0.03395, \quad \alpha_{3}\left(M_{Z}\right)^{e x p}=0.125
$$

Squark and slepton masses in our scenario are given (at $M_{S t r}$ ) by eq.(17) where, of course, a standard RG running is necessary in order to obtain the physical masses. Furthermore, for the left and right top-squarks there is a non-trivial mixing in the mass matrix which has to be conveniently taken into account. Nevertheless, as was mentioned in the previous section, gaugino masses in our framework tend to be clearly smaller than scalar masses, so the relevant experimental (lower) bounds for us are those corresponding to $M_{3}$ and $M_{\chi^{ \pm}}$. In particular, comparing the theoretical gluino mass (25) with the experimental limit (24) we obtain the following bound on $m_{3 / 2}, m_{\tilde{q}}, m_{\tilde{l}}$,

$$
\begin{aligned}
m_{3 / 2} & \gtrsim \frac{79 \mathrm{GeV}}{\alpha_{3}\left(M_{Z}\right)\left(0.0120 \delta^{G S}+0.0029\right)} \\
m_{\tilde{q}}\left(M_{S t r}\right), m_{\tilde{l}}\left(M_{S t r}\right) & \gtrsim(79 \mathrm{GeV}) \frac{\sqrt{1+\mathrm{n}_{\alpha}\left(0.078-1.3 \times 10^{-4} \delta^{\mathrm{GS}}\right)}}{\alpha_{3}\left(\mathrm{M}_{\mathrm{Z}}\right)\left(0.0120 \delta^{\mathrm{GS}}+0.0029\right)},
\end{aligned}
$$

where we have used eq.(17). Similar bounds, but involving also $\mu$ and $\sin 2 \beta$, are obtained from the lightest chargino mass (26).

Before going into the naturalness constraints, let us remark that there exits an upper bound on the value of $\left|A_{t}\right|$ (i.e. the parameter of the trilinear scalar term coming from the top Yukawa coupling) in order to prevent the scalar potential of developing colour and electric charge breaking minima. Assuming universality for the SUSY breaking scalar masses $\left(m_{\phi}=m\right)$, this bound reads $A_{t}^{2} \leq 3\left(3+\mu^{2} / m^{2}\right)$ at the GUT scale 31. Hence, 
for $\mu^{2}<<m^{2}$ (which will be the case here) we can simply use $\left|A_{t}\right| \leq 3$. This constraint is trivially satisfied if the top Yukawa coupling is of the untwisted type, see eq.(18). In general, $\left|A_{t}\right|$ is given by eq.(19) and is small enough to avoid the existence of these troublesome vacua.

\subsection{Naturalness constraints}

The naturalness constraints come from requiring the absence of fine tuning in the electroweak breaking mechanism. Let us review very briefly the previous work on this issue. The standard criterion [32 to avoid the fine tuning is to impose

$$
\left|\frac{a_{i}}{M_{Z}^{2}} \frac{\partial M_{Z}^{2}\left(a_{i}, h_{t}\right)}{\partial a_{i}}\right|<\Delta,
$$

where $a_{i}$ are the theoretical parameters defining the SUSY breaking, $h_{t}$ is the top Yukawa coupling, which is taken as an independent parameter, and $\Delta$ measures the allowed degree of fine tuning. In ref. 32 the soft breaking parameters $M, m, \mu, A, B$ were taken as the $a_{i}$ parameters of eq.(29), assuming universality of the SUSY breaking gaugino and scalar masses at the unification scale (i.e. $m_{\phi_{\alpha}} \equiv m, M_{a} \equiv M \forall a, \alpha$ ). The relevant expression for $M_{Z}^{2}$ is

$$
M_{Z}^{2}=\frac{2\left(\mu_{1}^{2}-\mu_{2}^{2} \operatorname{tg}^{2} \beta\right)}{\operatorname{tg}^{2} \beta-1},
$$

where the $\beta$ angle, defined in eq.(26), is given by $\sin 2 \beta=2 \mu_{3}^{2} /\left(\mu_{1}^{2}+\mu_{2}^{2}\right)$. This comes from minimizing the neutral part of the Higgs potential

$$
V\left(H_{1}, H_{2}\right)=\frac{1}{8}\left(g^{2}+g^{\prime 2}\right)\left(\left|H_{1}\right|^{2}-\left|H_{2}\right|^{2}\right)^{2}+\mu_{1}^{2}\left|H_{1}\right|^{2}+\mu_{2}^{2}\left|H_{2}\right|^{2}-\mu_{3}^{2}\left(H_{1} H_{2}+\text { h.c. }\right)
$$

where $\mu_{1}, \mu_{2}, \mu_{3}$ are related to the soft breaking parameters via well-known expressions 33] coming from integrating the corresponding RG equations. More precisely,

$$
\begin{aligned}
\mu_{1}^{2} & =m^{2}+\mu^{2} l(t)+M^{2} g(t) \\
\mu_{2}^{2} & =m^{2}\left(h(t)-k(t) A^{2}\right)+\mu^{2} l(t)+M^{2} e(t)+A m M f(t) \\
\mu_{3}^{2} & =-\mu m B q(t)+\mu M r(t)+A m \mu s(t)
\end{aligned}
$$

where $l(t), g(t)$, etc. are RG quantities whose explicit dependence on the RG parameter $t=2 \log \left(M_{G U T} / M_{Z}\right), \alpha_{a}$ and $h_{t}$ can be found in ref.[33], where the minimal particle content and the usual approximation $h_{b}, h_{\tau}<<h_{t}$ were used. Using eqs. (30) and (32) 
the naturalness conditions (29) for each soft breaking parameter are easily obtained [32]. A further simplification consists of working around the approximate zeros of eq.(30), i.e.

$$
\mu_{1}^{2} \mu_{2}^{2} \simeq \mu_{3}^{4}
$$

This is equivalent to requiring the right value for $M_{Z}$ in (30), neglecting $\frac{M_{Z}^{2}}{M^{2}}, \frac{M_{Z}^{2}}{m^{2}}, \frac{M_{Z}^{2}}{\mu^{2}}$ terms. Then the left hand side of eq.(29) is simplified since

$$
\frac{a_{i}}{M_{Z}^{2}} \frac{\partial M_{Z}^{2}\left(a_{i}, h_{t}\right)}{\partial a_{i}}=\frac{2\left(\partial / \partial a_{i}\right)\left(\mu_{1}^{2} \mu_{2}^{2}-\mu_{3}^{4}\right)}{M_{Z}^{2}\left(\mu_{1}^{2}-\mu_{2}^{2}\right)} .
$$

The scenario we are analysing is similar to the previous one, but presents some peculiarities. First, the RG running has to be made from $M_{S t r}$ instead of $M_{G U T}$. Likewise, neither the soft breaking parameters $m_{\phi_{\alpha}}^{2}, M_{a}$, nor the gauge couplings $\alpha_{a}$ are "unified" at $M_{S t r}$. More precisely, $m_{\phi_{\alpha}}^{2}$ and $M_{a}$ are given by eqs.(17,21), whereas $\alpha_{a}$ at $M_{S t r}$ should be obtained by inserting $\alpha_{a}^{e x p}\left(M_{Z}\right)$ in the corresponding RG equations, where we have taken the minimal particle content too. All this modifies the previous eqs.(32) in a straightforward way?. Second, in our framework $M_{a}, m_{\phi_{\alpha}}^{2}$ and $A$ are not independent parameters since they are related to $m_{3 / 2}$ and $\delta^{G S}$ through eqs.(17, 19, 21). In consequence, eqs.(32) become

$$
\begin{aligned}
\mu_{1}^{2} & =C_{1}\left(\delta^{G S}\right) m_{3 / 2}^{2}+D \mu^{2} \\
\mu_{2}^{2} & =C_{2}\left(\delta^{G S}\right) m_{3 / 2}^{2}+D \mu^{2} \\
\mu_{3}^{2} & =\mu m_{3 / 2}\left[C_{3}\left(\delta^{G S}\right)+C_{4} B\right],
\end{aligned}
$$

where the explicit expressions of $C_{i}, D$ can readily be obtained from (32) and (17, 19,21) in the above explained manner. For simplicity we have chosen here $H_{1}$ and $H_{2}$ as untwisted fields, thus $n_{H_{1}}=n_{H_{2}}=-1$ in eq.([17). Recall however that the actual dependence of $m_{\phi_{\alpha}}^{2}$ on $n_{\alpha}$ is very small. Moreover, condition (33) (which in this case amounts to neglect $\frac{M_{Z}^{2}}{m_{3 / 2}^{2}}, \frac{M_{Z}^{2}}{\mu^{2}}$ terms) reads

$$
\begin{aligned}
\left(\frac{\mu}{m_{3 / 2}}\right)^{2} & \simeq-\frac{1}{2 D^{2}}\left[D\left(C_{1}+C_{2}\right)-\left(C_{3}+C_{4} B\right)^{2}\right. \\
& \left. \pm \sqrt{\left(D\left(C_{1}+C_{2}\right)-\left(C_{3}+C_{4} B\right)^{2}\right)^{2}-4 D^{2} C_{1} C_{2}}\right] \equiv f(B)
\end{aligned}
$$

\footnotetext{
${ }^{5}$ The quantitative effect of these modifications, however, is very small.
} 
Clearly, the role of the independent $a_{i}$ parameters in (29) has to be played here by $m_{3 / 2}$, $\mu$ and $B$. Consequently, our naturalness conditions are

$$
\begin{array}{rll}
m_{3 / 2} & : \quad m_{3 / 2}^{2}<\frac{M_{Z}^{2}}{4}\left|\frac{C_{1}-C_{2}}{2 C_{1} C_{2}+f(B)\left(D\left(C_{1}+C_{2}\right)-\left(C_{3}+C_{4} B\right)^{2}\right)}\right| \Delta \\
\mu & : \quad \mu^{2}<\frac{M_{Z}^{2}}{4}\left|\frac{C_{1}-C_{2}}{2 D^{2} f(B)+D\left(C_{1}+C_{2}\right)-\left(C_{3}+C_{4} B\right)^{2}}\right| \Delta \\
B: & \left|B\left(C_{3}+C_{4} B\right) \mu^{2}\right|<\frac{M_{Z}^{2}}{4}\left|\frac{C_{1}-C_{2}}{C_{4}}\right| \Delta .
\end{array}
$$

In writing(37) and (38) $\mu^{2}$ and $m_{3 / 2}^{2}$ have respectively been eliminated through (36). Of course, the level of stringency of conditions (37 39) depends on the value of $\Delta$, which is, to some extent, arbitrary. More precisely, the bounds on $m_{3 / 2}$ and $\mu$ (and hence those on $M_{a}$ and $m_{\tilde{q}}$ ) scale as $\sqrt{\Delta}$. Notice also that if we had taken $M_{Z}$ instead of $M_{Z}^{2}$ in eq. (29), then the bounds would have been less restrictive by a factor of $\sqrt{2}$. Therefore, we have adopted a rather conservative point of view, taking $\Delta=50$. Following ref. 32. the quantitative results are conveniently given in terms of the quantity $M_{t}$, defined as

$$
M_{t}=\frac{\sqrt{2}}{g_{2}} M_{W} h_{t},
$$

so that the physical top mass is given by

$$
m_{t}=M_{t} \sin \beta
$$

The positivity of the right hand side of eq.(36), selects a definite sign of the square root and, together with the naturalness conditions (37 39), imposes a lower bound on $M_{t}$, say $M_{t}^{\min }$. This corresponds to the value of $M_{t}^{\text {min }}$ for which the quantity $C_{2}$ is vanishing, which depends also on the value of $A$. This is shown in fig.1: maintaining $|A| \leq 3, M_{t}^{\text {min }}$ is forced to be in the region

$$
80 \mathrm{GeV} \leq \mathrm{M}_{\mathrm{t}}^{\min } \leq 158 \mathrm{GeV}
$$

where the upper limit corresponds to the untwisted case. The naturalness conditions (37 39) for a typical case $\left(\delta^{G S}=45\right)$ are illustrated in fig.2. More precisely, in fig.2a the upper bounds on $m_{3 / 2}$ and $\mu$, i.e. eqs.(37,38), have been represented as functions of $M_{t}-M_{t}^{\text {min }}$. [The thickness of the curves simply counts the variation of $B$ within its

\footnotetext{
${ }^{6}$ This kind of limit did not appear in ref. 32, since the value of $B$ was always taken such that eq. (33) were fulfilled, without taking into account the naturalness bounds for it.
} 
naturalness limits, given by (39)]. This is translated into upper bounds on $M_{3}, M_{\chi^{ \pm}}$ through eqs.(25.26), which is illustrated in fig.2b. We have also represented the lower experimental bounds on $M_{3}, M_{\chi^{ \pm}}$(dashed lines), so that the complete allowed range of variation for $M_{3}, M_{\chi^{ \pm}}$and $M_{t}$ can be easily observed. Analogously, upper bounds on squark and slepton masses are obtained through eq.(17). Note that this equation gives the masses at $M_{S t r}$, so a standard RG running has been performed. The corresponding upper bounds are represented in fig.2c. Just for simplicity we have considered here the case of untwisted fields [i.e. $n_{\alpha}=-1$ in eq.(17)], but the extrapolation to the general case is straightforward. As was mentioned above, it is clear that the experimental lower bounds on squark and slepton masses do not play any role since, once $M_{3}, M_{\chi^{ \pm}}$are compelled to respect their experimental lower bounds, the former are automatically fulfilled thanks to eq.(28). In consequence, the relevant lower bound for $m_{\tilde{q}}, m_{\tilde{l}}$ is the one coming from eq.(28), which has been included in the figure (dashed line). Note that this bound arises from the experimental gluino limit [see eq.(24)]. The one corresponding to the chargino (which has not been represented) is a bit more stringent, although it is subjected to the above mentioned condition on the lightest neutralino mass. Notice also that indeed $\mu^{2}<<m_{\phi}^{2}$, as is required for the approximation $|A| \leq 3$. Let us summarize the allowed ranges for the relevant masses in the $\delta^{G S}=45$ case. Excluding values of $M_{t}$ very close to the peak in fig.2 (which would amount to a new fine tuning by itselfi]) these are

$$
\begin{aligned}
& 1100 \mathrm{GeV} \leq \mathrm{m}_{3 / 2} \leq 4200 \mathrm{GeV}, \quad 350 \mathrm{GeV} \leq \mu \leq 450 \mathrm{GeV} \\
& 79 \mathrm{GeV} \leq \mathrm{M}_{3} \leq 285 \mathrm{GeV}, \quad 22 \mathrm{GeV} \leq \mathrm{M}_{\chi^{ \pm}} \leq 80 \mathrm{GeV} \\
&-7 \mathrm{GeV} \leq \mathrm{M}_{\mathrm{t}}^{\min }-\mathrm{m}_{\mathrm{t}} \leq 1 \mathrm{GeV} \quad, \quad 1070 \mathrm{GeV} \leq \mathrm{m}_{\tilde{\mathrm{q}}}, \mathrm{m}_{\tilde{\mathrm{I}}} \leq 4080 \mathrm{GeV},
\end{aligned}
$$

where $M_{t}^{\min }$ is constrained by eq.(42). All the lower bounds in (43) arise from the experimental gluino limit in eq.(24). The previous allowed ranges are narrowing as $\delta^{G S}$ decreases, so that for $\delta^{G S}<25$ they virtually disappear. Therefore, we conclude that $\delta^{G S}>25$ is a necessary condition for respecting all the (experimental and naturalness) bounds on supersymmetric masses. It is remarkable in (43) the smallness of the gaugino masses (quite close to their experimental lower limits of eq.(24)) compared with the squark and slepton masses. Furthermore, the top mass $m_{t}$ (see eqs.(43,42)) is notably consistent with its present experimental bounds. Finally, let us recall that the values in eq. (43) have been obtained by using eq.(21) for the gaugino masses, which is strictly valid only for the $Z_{3}, Z_{7}$ orbifolds. Therefore, as was mentioned in the text, the previous

\footnotetext{
${ }^{7}$ More precisely, we are allowing for a fine tuning of $0.5 \mathrm{GeV}$ in the value of $M_{t}$.
} 
numbers can oscillate within a 30\% error in the general case. This does not substantially modify, however, the main conclusions.

\section{$5 \quad$ Summary and conclusions}

We have calculated the form of the soft SUSY breaking terms arising from a large class of string models, more precisely symmetric orbifold constructions, and studied its phenomenological viability. The form of the soft breaking terms depends on certain parameters, such as the value of $\delta^{G S}$ (the contribution of the Green-Schwarz mechanism to the gauge kinetic function) and the modular weights of the relevant superfields. In the calculation the cosmological constant has been assumed to vanish. A first conclusion is that some of the common assumptions of the minimal supersymmetric standard model, in particular universality for all the gaugino masses, scalar masses and trilinear terms, do not hold in general (this was also noted in ref.22]).

In order to get quantitative results a specific SUSY breaking mechanism has to be considered. In this sense the only mechanism so far analysed, which is capable of generating a hierarchical SUSY breakdown in string constructions, is gaugino condensation in the hidden sector. Assuming this scenario, we have given some explicit numerical formulae for the various soft breaking terms. At this point it becomes clear that squark and slepton masses tend to be much larger than gaugino masses.

Finally, we have studied the phenomenological viability of the soft breaking terms. There are two types of tests that they must pass. First, they have to be consistent with the experimental (lower) bounds on gaugino masses, squark masses, etc. Second, they should be small enough not to spoil the SUSY solution to the gauge hierarchy problem, guaranteeing a successful $S U(2) \times U(1)_{Y} \rightarrow U(1)_{e m}$ breaking without the need of fine tuning. We have found that the considered scenarios can be perfectly consistent with both tests. This is certainly non-trivial and in fact imposes interesting constraints on measurable quantities (e.g. gaugino and scalar masses). These constraints are summarized in the allowed ranges of eq. (43) for the $\delta^{G S}=45$ case. These are narrowing as $\delta^{G S}$ decreases, so that for $\delta^{G S}<25$ they virtually disappear. From eqs.(43.24) it is clear that gluino and chargino masses should not be much higher than their present experimental lower bounds, while squark and slepton masses must be much larger. All this can be considered as an experimental signature of these stringy scenarios. Also, the top mass is constrained to be within a range [see eqs.(43,44)] remarkably consistent with its present experimental bounds. 


\section{ACKNOWLEDGEMENTS}

We thank L.E. Ibáñez for very useful comments and suggestions. The work of B.C. was supported by a Comunidad de Madrid grant.

\section{References}

[1] L. Dixon, talk presented at the A.P.S. D.P.F. Meeting at Houston (1990); V. Kaplunovsky, talk presented at the "Strings 90" workshop at College Station (1990); L. Dixon, V. Kaplunovsky, J. Louis and M. Peskin, unpublished

[2] J.A. Casas, Z. Lalak, C. Muñoz and G.G. Ross, Nucl. Phys. B347 (1990) 243

[3] A. Font, L. Ibáñez, D. Lüst and F. Quevedo, Phys. Lett. B245 (1990) 401

[4] S. Ferrara, N. Magnoli, T.R. Taylor and G. Veneziano, Phys. Lett. B245 (1990) 409

[5] H.P. Nilles and M. Olechowsky, Phys. Lett. B248 (1990) 268; P. Binétruy and M.K. Gaillard, Phys. Lett. B253 (1991) 119

[6] M. Cvetič, A. Font, L. Ibáñez, D. Lüst and F. Quevedo, Nucl. Phys. B361 (1991) 194

[7] D. Lüst and T.R. Taylor, Phys. Lett. B253 (1991) 335

[8] B. de Carlos, J.A. Casas and C. Muñoz, Phys. Lett. B263 (1991) 248

[9] J. Louis, talk presented at the Particles and Fields'91 Symposium, Vancouver (1991)

[10] D. Lüst and C. Muñoz, Phys. Lett. B279 (1992) 272

[11] B. de Carlos, J.A. Casas and C. Muñoz, CERN-TH.6436/92 (1992)

[12] L. Dixon, J. Harvey, C. Vafa and E. Witten, Nucl. Phys. B261 (1985) 651, B274 (1986) 285

[13] J.A. Casas, E.K. Katehou and C. Muñoz, Nucl. Phys. B317 (1989); J.A. Casas and C. Muñoz, Phys. Lett. B214 (1988) 63; A. Font, L. Ibáñez, H.P. Nilles and F. Quevedo, Phys. Lett. B210 (1988) 101 
[14] V. Kaplunovsky, Nucl. Phys. B307 (1988) 145; L. Dixon, V. Kaplunovsky and J. Louis, Nucl. Phys. B355 (1991) 649

[15] J.P. Derendinger, S. Ferrara, C. Kounnas and F. Zwirner, Nucl. Phys. B372 (1992) 145

[16] J. Louis, talk given at the 2nd International Symposium on Particles, Strings and Cosmology, Boston (1991); G. Lopes Cardoso and B. Ovrut, Nucl. Phys. B369 (1992) 351

[17] L. Dixon, V. Kaplunovsky and J. Louis, Nucl. Phys. B329 (1990) 27

[18] E. Witten, Phys. Lett. B155 (1985) 151; S. Ferrara, C. Kounnas and M. Porrati, Phys. Lett. B181 (1986) 263; M. Cvetič, J. Louis and B. Ovrut, Phys. Lett. B206 (1988) 227

[19] S. Ferrara, D. Lüst and S. Theisen, Phys. Lett. B233 (1989) 147

[20] S. Hamidi and C. Vafa, Nucl. Phys. B279 (1987) 465; L. Dixon, D. Friedan, E. Martinec and S. Shenker, Nucl. Phys. B282 (1987) 13; T.T. Burwick, R.K. Kaiser and H.F. Müller, Nucl. Phys. B355 (1991) 689; T. Kobayashi and N. Ohtsubo, DPKU9103; J.A. Casas, F. Gómez and C. Muñoz, CERN-TH.6194/91 (1991), to appear in Int. J. Mod. Phys. A

[21] J. Louis, private communication

[22] L.E. Ibáñez and D. Lüst, Nucl. Phys. B382 (1992) 305

[23] E. Cremmer, S. Ferrara, L. Girardello and A. Van Proeyen, Nucl. Phys. B212 (1983) 413

[24] J.E. Kim and H.P. Nilles, Phys. Lett. B138 (1984) 150; G.F. Giudice and A. Masiero, Phys. Lett. B206 (1988) 480; J.E. Kim and H.P. Nilles, Phys. Lett. B263 (1991) 79; E.J. Chun, J.E. Kim and H.P. Nilles, Nucl. Phys. B370 (1992) 105.

[25] H.P. Nilles, M. Srednicki and D. Wyler, Phys. Lett. B120 (1983) 346; J.M. Frere, D.R.T. Jones and S. Raby, Nucl. Phys. B222 (1983) 11; J.P. Derendinger and C. Savoy, Nucl. Phys. B237 (1984) 307; J. Ellis, J.F. Gunion, H.E. Haber, L. Roszkowski and F. Zwirner, Phys. Rev. D39 (1989) 844; M. Drees, Int. J. Mod. Phys. A4 (1989) 3635; J.R. Espinosa and M. Quirós, Phys. Lett. B279 (1992) 92, IEM-FT-60/92 (1992) 
[26] H.P. Nilles, Phys. Lett. B115 (1982) 193; S. Ferrara, L. Girardello and H.P. Nilles, Phys. Lett. B125 (1983) 457; J.P. Derendinger, L.E. Ibáñez and H.P. Nilles, Phys. Lett. B155 (1985) 65; M. Dine, R. Rohm, N. Seiberg and E. Witten, Phys. Lett. B156 (1985) 55; C. Kounnas and M. Porrati, Phys. Lett. B191 (1987) 91

[27] N.V. Krasnikov, Phys. Lett. B193 (1987) 37

[28] J.A. Casas, F. Gómez and C. Muñoz, CERN-TH.6507/92 (1992), to appear in Phys. Lett. B

[29] Particle Data Group, Phys. Rev. D45 (1992) 1

[30] See for example F. Zwirner, talk presented at the Workshop on Physics and Experiments with Linear Colliders, Saariselkä, Finland (1991)

[31] C. Kounnas, A.B. Lahanas, D.V. Nanopoulos and M. Quirós, Nucl. Phys. B236 (1984) 438; J.P. Derendinger and C. Savoy, Nucl. Phys. B237 (1984) 307

[32] R. Barbieri and G.F. Giudice, Nucl. Phys. B306 (1988) 63

[33] L.E. Ibáñez, C. López and C. Muñoz, Nucl. Phys. B256 (1985) 218

\section{FIGURE CAPTIONS}

Fig.1 $M_{t}^{\min }$ (in $\mathrm{GeV}$ ) versus $A$.

Fig.2a Upper bounds on $m_{3 / 2}, \mu$ (in GeV) versus $M_{t}-M_{t}^{\text {min }}$ (in $\mathrm{GeV}$ ). The thickness of the curves counts the variation of $B$ within its naturalness limits.

Fig.2b Upper bounds on the gluino mass $\left(M_{3}\right)$ and the chargino mass $\left(M_{\chi^{ \pm}}\right)$(in $\mathrm{GeV}$ ) versus $M_{t}-M_{t}^{\min }$ (in $\mathrm{GeV}$ ). The dashed lines represent the corresponding lower experimental bounds. The one for $M_{\chi^{ \pm}}$applies only if the lightest neutralino mass is smaller than $28 \mathrm{GeV}$. The thickness of the curves counts the variation of $B$ within its naturalness limits.

Fig.2c The same as in fig.2b but for the squark and slepton masses $\left(m_{\tilde{q}}, m_{\tilde{l}}\right)$. The dashed line represents the lower bound coming from the experimental bound on the gluino mass (see eq.(28)). 